Pacific Journal of Mathematics

ON THE ASSOCIATIVITY AND COMMUTATIVITY OF 


\title{
ON THE ASSOCIATIVITY AND COMMUTATIVITY OF ALGEBRAS OVER COMMUTATIVE RINGS
}

\author{
Kwangil Koh, Jiang Luh and Mohan S. Putcha
}

\begin{abstract}
Let $A$ be an algebra (not necessarily associative) over a commutative ring $R$. $A$ is left scalar associative if for each $a, b, c \in A$ there exists $\alpha \in R$ depending on $a, b, c$ such that $(a b) c=\alpha a(b c)$. A right scalar associativity is defined similarly. $A$ is scalar commutative if for each $a, b$ in $A$, there exists $\alpha \in R$ depending on $a, b$ such that $\alpha a b=b a$. In this paper, it is shown that if $A$ is right and left scalar associative and scalar commutative then $(a b) c-a(b c)$ and $a b-b a$ are nilpotent for every $a, b$ and $c$ in $A$. If $1 \in A$, then $[(a b) c-a(b c)]^{2}=0$. If $R$ is a principal ideal domain then $A$ is associative and commutative.
\end{abstract}

Introduction. Coughlin and Rich [1] and Coughlin, Kleinfeld and Rich [2] have studied algebras $A$ over a field $F$ with the property that for any $x, y, z \in A$ there exists $\alpha \in F$ depending on $x, y, z$ such that $(x y) z=\alpha x(y z)$. They show that if $A$ has a nonzero idempotent then this condition implies associativity. Rich [4] has shown that if for each $x, y \in A$ there exists $\alpha \in F$ depending on $x, y$ such that $x y=\alpha y x$, then $A$ is either commutative or anti-commutative. In this paper we study related conditions for an algebra $A$ over a comutative ring $R$. If either $A$ has no zero divisors or if $A$ has identity element and $R$ is a principal ideal domain, then we can still prove associativity and commutativity under the respective conditions. In general with some additional minor constraints we are able to prove the nilpotency of associators and commutators in $A$.

1. Preliminaries. Throughout this paper $N$ will denote the set of natural numbers and $Z^{+}$will denote the set of positive integers. $R$ will denote a commutative, associative ring which may or may not have an identity element. $A$ will denote a not necessarily associative ring which may or may not have an identity element. We assume that $A$ is an algebra over $R$ in the sense that for all $a, b \in A$ and $\alpha, \beta \in R,(\alpha+\beta) \alpha=\alpha a+\beta a, \alpha(a+b)=\alpha a+\alpha b,(\alpha \beta) a=$ $\alpha(\beta a)$, and $\alpha(a b)=(\alpha a) b=a(\alpha b)$. As usual if $a, b, c \in A$, the associator $(a, b, c)=(a b) c-a(b c)$ and the commutator $[a, b]=a b-b a$. We will be concerned with the following generalizations of concepts introduced in [1], [4].

Definition 1. $A$ is left scalar associative if for each $a, b, c \in A$ there exists $\alpha \in R$ depending on $a, b, c$ such that $(a b) c=\alpha a(b c)$. 
2. $A$ is right scalar associative if for each $a, b, c \in A$ there exists $\alpha \in R$ depending on $a, b, c$ such that $a(b c)=\alpha(a b) c$.

3. $A$ is scalar associative if it is both right and left scalar associative.

4. $A$ is scalar commutative if for each $a, b \in A$ there exists $\alpha \in R$ depending on $a, b$ such that $a b=\alpha b a$.

LEMMa 1.1. (i) If $A$ is scalar associative, then for all $a, b, c \in$ $A, \alpha \in R, \alpha a(b c)=0$ if and only if $\alpha(a b) c=0 . \quad$ Also, $(a b) c=0$ if and only if $a(b c)=0$.

(ii) If $A$ is scalar commutative, then for all $a, b \in A, \alpha \in R$, $\alpha a b=0$ if and only if $\alpha b a=0$. Also, $a b=0$ if and only if $b a=0$.

LEMMA 1.2. (i) Suppose $A$ is scalar associative. Let $x, y, z, u \in$ $A, \alpha, \beta \in R$ such that $(x, y, u)=0,(x y) z=\alpha x(y z)$ and $x(y(z+u))=$ $\beta(x y)(z+u)$. Then $x y u-\alpha x y u-\beta x y u+\alpha \beta x y u=0$.

(ii) Suppose $A$ is scalar commutative. Let $x, y, u \in A, \alpha, \beta \in R$ such that $y u=u y, y x=\alpha x y$ and $(x+u) y=\beta y(x+u)$. Then $y u-$ $\alpha y u-\beta y u+\alpha \beta y u=0$.

Proof. (i) We have $x(y(z+u-\alpha \beta z-\beta u))=0$ and hence by Lemma 1.1, $(x y)(z+u-\alpha \beta z-\beta u)=0$. Also $\alpha \beta(x y)(z+u)=(x y) z+$ $\alpha x y u$. Combining these we have the result.

(ii) We have $(x+u-\alpha \beta x-\beta u) y=0$. Hence by Lemma 1.1, $y(x+u-\alpha \beta x-\beta u)=0$. Also $\alpha \beta y(x+u)=y x+\alpha y u$. Combining these we have the result.

2. Associators and commutators. The main purpose of this section is to study nilpotency of associators and commutators.

Theorem 2.1. Let $A$ be scalar commutative. If either $A$ has an identity element or is scalar associative, then the square of every commutator in $A$ is zero.

Proof. Let $x, y \in A$. There exists $\alpha \in R$ such that $y x=\alpha x y$. First assume $A$ has an identity element 1 . Then there exists $\beta \in R$ such that $(x+1) y=\beta y(x+1)$. By Lemma 1.2 we set $y-\alpha y-\beta y+$ $\alpha \beta y=0$. Hence $y(x+1)-\alpha y(x+1)=\beta y(x+1)-\alpha \beta y(x+1)=$ $(x+1) y-\alpha(x+1) y$. This implies that $(x y-y x)^{2}=(x y-\alpha x y)^{2}=0$.

Next assume $A$ is scalar associative. There exists $\gamma \in R$ such that $(x+y) y=\gamma y(x+y)$. By Lemma 1.2, $y^{2}-\alpha y^{2}=\gamma y^{2}-\alpha \gamma y^{2}$. Multiplying the first equation by $\alpha$ and then subtracting from itself, we get $x y-\alpha x y=\gamma y x-\alpha \gamma y x$. Now there exists $\delta \in R$ such 
that $y(y x)=\delta y^{2} x$. So $y(x y-\alpha x y)=\gamma y(y x)-\alpha \gamma y(y x)=\delta\left(\gamma y^{2}-\alpha \gamma y^{2}\right) x=$ $\delta\left(y^{2}-\alpha y^{2}\right) x=y(y x)+\alpha y(y x)=\alpha y(\gamma y)-\alpha^{2} y(x y)$. Hence $y(x y-2 \alpha x y+$ $\left.\alpha^{2} x y\right)=0$. By Lemma 1.1, $(x y-y x)^{2}=(x y-\alpha x y)^{2}=0$.

Theorem 2.2. Suppose $A$ has an identity element 1 and is scalar associative. Then the square of every associator in $A$ is zero.

Proof. Let $x, y, z \in A$. There exist $\alpha, \beta \in R$ such that $(x y) z=$ $\alpha x(y z)$ and $x(y(z+1))=\beta(x y)(z+1)$. By Lemma 1.2, $x y-\alpha x y=$ $\beta x y-\alpha \beta y$. So

$$
\begin{aligned}
(x y)(z+1)-\alpha(x y)(z+1) & =\beta(x y)(z+1)-\alpha \beta(x y)(z+1) \\
& =x(y(z+1))-\alpha x(y(z+1)) .
\end{aligned}
$$

Thus $(x, y, z)^{2}=0$.

THEOREM 2.3. Suppose A has no zero divisors.

(i) If $A$ is scalar commutative then $A$ is commutative.

(ii) If $A$ is scalar associative then $A$ is associative.

Proof. (i) Let $x, y \in A$. There exist $\alpha, \beta \in R$ such that $y x=$ $\alpha x y$ and $(x+y) y=\beta y(x+y)$. By Lemma 1.2, $(y-\alpha y)(y-\beta y)=0$. So $y=\alpha y$ or $y=\beta y$. In either case $x y=y x$.

(ii) Let $u, v, w \in A$ be nonzero. Let $\alpha \in R$ be such that $u v=$ $\alpha u(v w)$. Let $w^{\prime} \in A$. We make the following claim.

$$
\text { If }\left[w, w^{\prime}\right] \neq 0 \text {, then }(u v) w^{\prime}=\alpha u\left(v w^{\prime}\right) .
$$

There exists $\gamma \in R$ such that $(u v) w^{\prime}=\gamma u\left(v w^{\prime}\right)$. Suppose (1) is not true. Then $\alpha u \neq \gamma u$. There exists $\beta \in R$ such that $(u v)\left(w+w^{\prime}\right)=$ $\beta u\left(v\left(w+w^{\prime}\right)\right)$. So $\alpha u(v w)+\gamma u\left(v w^{\prime}\right)=\beta u(v w)+\beta u\left(v w^{\prime}\right)$. Since $A$ has no zero divisors we get $\alpha w+\gamma w^{\prime}=\beta w+\beta w^{\prime}$. Hence $(\alpha-\beta) w=$ $(\beta-\gamma) w^{\prime}$. Thus $w^{\prime}$ commutes with $(\alpha-\beta) w$ and $w$ commutes with $(\beta-\gamma) w^{\prime}$. So,

$$
\begin{aligned}
& (\alpha-\beta)\left[w, w^{\prime}\right]=\left[(\alpha-\beta) w, w^{\prime}\right]=0 \\
& (\beta-\gamma)\left[w^{\prime}, w\right]=\left[(\beta-\gamma) w^{\prime}, w\right]=0
\end{aligned}
$$

Hence

$$
(\alpha-\beta) u\left[w, w^{\prime}\right]=0=(\beta-\gamma) u\left[w, w^{\prime}\right] .
$$

Since $\left[w, w^{\prime}\right] \neq 0$ we get $(\alpha-\beta) u=0=(\beta-\gamma) u$. Hence $\alpha u=\gamma u$, a contradiction. So (1) is true. Similarly, it can be seen that if $u^{\prime}, v^{\prime} \in A$, then 


$$
\begin{aligned}
{\left[u, u^{\prime}\right] } & \neq 0 \text { implies }\left(u^{\prime} v\right) w=\alpha u^{\prime}(v w) \\
{\left[v, v^{\prime}\right] \neq 0 \text { implies }\left(u v^{\prime}\right) w } & =\alpha u\left(v^{\prime} w\right)
\end{aligned}
$$

Next we show that for all $x \in A, x \cdot x^{2}=x^{2} \cdot x$. Suppose not. Then there exists $x \in A$ such that $\left[x, x^{2}\right] \neq 0$. So $x \neq 0$ and there exists $\delta \in R$ such that $(x \cdot x) x=\delta x(x \cdot x)$. By (1), (2) we get $\left(x^{2} \cdot x\right) x=\delta x^{2}(x \cdot x)$, $\left(x \cdot x^{2}\right) x=\delta x\left(x^{2} \cdot x\right)$ and $(x \cdot x) x^{2}=\delta x\left(x \cdot x^{2}\right)$. So $\left(x^{2} \cdot x\right) x=\delta x^{2}(x \cdot x)=$ $\delta(x \cdot x) x^{2}=\delta^{2} x\left(x \cdot x^{2}\right)=\delta x\left(\delta x \cdot x^{2}\right)=\delta x\left(x^{2} \cdot x\right)=\left(x \cdot x^{2}\right) x$. This contradiction shows that $x^{2} \cdot x=x \cdot x^{2}$ for all $x \in A$. Now let $a, b, c \in A, a, b, c \neq 0$ such that $(a, b, c)=0$. Let $c^{\prime} \in A$. There exist $\mu, \nu \in R$ such that $(a b) c^{\prime}=\mu a\left(b c^{\prime}\right), a\left(b\left(c^{\prime}+c\right)\right)=\nu(a b)\left(c^{\prime}+c\right)$. By Lemma 1.2 and the fact that $A$ is a domain we get $(a-\mu a)(a-\nu a)=0$. So $a=\mu a$ or $a=\nu a$. In either case $\left(a, b, c^{\prime}\right)=0$. By duality we can therefore conclude that for $a, b, c \neq 0,(a, b, c)=0$ implies that $\left(a^{\prime}, b, c\right)=\left(a, b^{\prime}, c\right)=$ $\left(a, b, c^{\prime}\right)=0$ for all $a^{\prime}, b^{\prime}, c^{\prime} \in A$. Starting with the fact that $x \cdot x^{2}=$ $x^{2} \cdot x$ for all $x \in A$, we can use the above repeatedly to conclude that $A$ is associative.

If $I$ is an ideal of $A$ which is also a subalgebra, then $A / I$ is also an algebra over $R$. If $A$ is scalar associative (resp. scalar commutative) then so is $A / I$. If $A$ is scalar associative and $x \in A$, then by Lemma 1.1, the $n$th power of $x$ is zero in one association if and only if it is zero in every association. Hence it makes sense to talk about nilpotent elements in $A$.

THEOREM 2.4. Let $A$ be scalar associative and scalar commutative. Then every associator and every commutator in $A$ is nilpotent.

Proof. The hypothesis implies that the nilpotents of $A$ form an ideal and a subalgebra. So without loss of generality, let $A$ have no nilpotent elements. By Theorem 2.1, $A$ is commutative. Call an ideal $I$ of $A$ prime if $a b \in I$ implies $a \in I$ or $b \in I$. We now follow some well known ideas (cf. [3; Chapter 4]). Let $x \in A, x \neq 0$. Let $T$ be the groupoid generated by $x$. Then $0 \notin T$. By Zorn's lemma there exists a maximal ideal $P$ of $A$ not intersecting $T$. We claim that $P$ is prime. Suppose there exist $a, b \in A$ such that $a, b \notin P$ but $a b \in P$. Let $P_{1}=\{n a+r a+u \mid n \in N, r \in A, u \in P\}$. Then by commutativity and scalar associativity, $P_{1}$ is an ideal of $A$ containing $P$ and $a$. Hence $P \cap T \neq \varnothing$. So there exists $d_{1}=n_{1} a+r_{1} a \in T$ for some $n_{1} \in N, r_{1} \in A$. Similarly there exists $d_{2}=n_{2} b+r_{2} b \in T$ for some $n_{2} \in N, r_{2} \in A$. So $d_{1} d_{2} \in T$. By commutativity, scalar associativity and the fact that $a b \in P$, we get $d_{1} d_{1} \in P$. This contradiction shows that $P$ is a prime ideal. Hence the intersection of all prime ideals is zero. We claim that each prime ideal $P$ is a subalgebra. For let $x \in P$. Then for any $\alpha \in R,(\alpha x)(\alpha x)=\left(\alpha^{2} x\right) \cdot x \in P$. Hence $\alpha x \in P$. 
Thus $A$ is a subdirect sum of algebras without zero divisors, each of which scalar associative. By Theorem 2.3, $A$ is associative.

3. Algebras with identity elements. Throughout this section we will assume that $R$ has an identity element 1 satisfying $1 \cdot a=a$ for all $a \in A$ and that $A$ itself has an identity element which will also be denoted by 1 .

LEMma 3.1. A is right scalar associative if and only if $A$ is left scalar associative if and only if $A$ is scalar associative.

Proof. By the dual nature of the conditions we may assume $A$ is left scalar associative and prove that $A$ is right scalar associative. Since $A$ is left scalar associative we have that for any $x, y, z \in A$, $x(y z)=0$ implies $(x y) z=0$. Now let $a, b, c \in A$. There exist $\alpha, \beta \in$ $R$ such that $(a b) c=\alpha a(b c)$, and $((a+1) b) c=\beta(a+1)(b c)$. Hence $\alpha a(b c)+b c=\beta a(b c)+\beta b c$. Thus $(\alpha a+(1-\beta) \cdot 1-\beta a)(b c)=0$. So $((\alpha a+(1-\beta) \cdot 1-\beta a) b) c=0$. It follows that $(\alpha-\beta)(a b) c=(\beta-1) b c=$ $(\alpha-\beta) a(b c)$. So $(\beta-1) \alpha(b c)=(\alpha-\beta) \alpha a(b c)=(\alpha-\beta)(a b) c=(\beta-1) b c$. Hence $(\beta-1)(a+1)(b c)=a[(\beta-1) b c]+(\beta-1) b c=a[(\beta-1) \alpha b c]+$ $(\beta-1) b c=(\beta-1) \alpha a(b c)+(\beta-1) b c=(\beta-1)(a b) c+(\beta-1) b c=$ $(\beta-1)((a+1) b) c$. Consequently, $((a+1) b) c=\beta(a+1)(b c)=(\beta-1)$. $(a+1)(b c)+(a+1)(b c)=(\beta-1)((a+1) b) c+(a+1)(b c)$. Thus $(a+1)(b c)=(2-\beta)((a+1) b) c$. Since $a, b, c$ are arbitrary we obtain that $A$ is right scalar associative.

Suppose $R$ is a P.I.D. (Principal Ideal Domain) and $a \in A$. Then we define order of $a, o(a)$, to be the generator of the ideal $I=$ $\{\alpha \mid \alpha \in R, \alpha a=0\}$ of $R$. Thus $o(\alpha)$ is unique up to associates in $R$. $o(a)=1$ if and only if $a=0$.

Lemma 3.2. Suppose $R$ is a P.I.D.

(i) If $A$ is scalar associative, $a, b \in R$ and $o(a b)=0$ then $(a, b, c)=0$ for all $c \in A$.

(ii) If $A$ is scalar commutative, $b \in R$ and $o(b)=0$ then $b$ is in the center of $A$.

Proof. (i) Let $c \in A$. There exist $\alpha, \beta \in R$ such that $(a b) c=$ $\alpha a(b c)$ and $a(b(c+1))=\beta(a b)(c+1)$. By Lemma 1.2, $(1-\alpha)(1-\beta) a b=$ 0 . So $\alpha=1$ or $\beta=1$ and thus $(a, b, c)=0$.

(ii) Let $b \in A$. There exist $\alpha, \beta \in R$ such that $b a=\alpha a b$ and $(\alpha+1) b=\beta b(\alpha+1)$. So by Lemma 1.2, $(1-\alpha)(1-\beta) b=0$. Hence $\alpha=1$ or $b=1$ and thus $a b=b a$.

Lemma 3.3. Suppose $R$ is a P.I.D. and $A$ is scalar associative. 
Assume further that there exists a prime $p \in R, m \in Z^{+}$such that $p^{m} A=0$. Then $A$ is associative.

Proof. Let $\mathscr{A}_{1}=\{(x, y) \mid x, y \in A$ and $(x, y, z)=0$ for all $z \in A\}$, $\mathscr{A}_{2}=\{(x, y) \mid x, y \in A$ and $(u, x, y)=0$ for all $u \in A\}$ and $\mathscr{A}=\mathscr{A}_{1} \cap$ $\mathscr{A}_{2}$. Let $x, y \in A$. Let $o(x y)=p^{k}, k \in N$. We prove by induction on $k$ that $(x, y) \in \mathscr{A}$. If $k=0$ then $x y=0$ and by Lemma $1.1,(x, y) \in$ A. So we assume $k>0$ and that the statement is true for $l<k$. We first show that for any $z \in A,(x, y, z) \neq 0$ implies $(x, y z, w)=0$ for all $w \in A$. So let $(x, y, z) \neq 0$. There exist $\alpha, \beta \in R$ such that $(x y) z=\alpha x(y z)$ and $((x+1) y) z=\beta(x+1)(y z)$. So $\alpha x(y z)+y z=\beta x(y z)+$

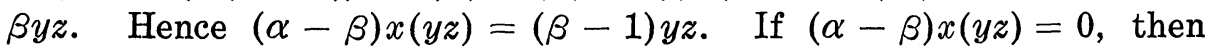
$\beta y z=y z$ whence $(x, y, z)=0$, a contradiction. So $(\alpha-\beta) x(y z) \neq 0$. In particular $\alpha-\beta \neq 0$ and $\alpha-\beta=\delta p^{t}$ for some $t \in N, \delta \in R,(\delta, p)=1$. If $t \geqq k$ then since $o(x y)=p^{k}$ we would get $(\alpha-\beta) x y=0$. But then $(\alpha-\beta)(x y) z=0$ which by Lemma 1.1 implies $(\alpha-\beta) x(y z)=0$, a contradiction. Hence $t<k$. Now since $p^{k}(x y) z=0$ we get $p^{k} x(y z)=$ 0 . So $p^{k-t}(\beta-1) y z=p^{k} \delta x(y z)=0$. Let $o(y z)=p^{i}$. We claim that $i \geqq k$. For suppose $i<k$. Then by induction hypothesis $(y, z) \in$ $\mathscr{A} \subseteq \mathscr{A}_{2}$. In particular $(x, y, z)=0$, a contradiction. So $i \geqq k$. Hence $p^{k}\left|p^{i}\right| p^{k-t}(\beta-1)$. Thus $p^{t} \mid \beta-1$ and $\beta-1=p^{t} \gamma$ for some $\gamma \in R$. So $p^{t}[\delta x(y z)-\gamma y z]=0$. Therefore $p^{t}[(\delta x-\gamma \cdot 1)(y z)]=0$. By induction hypothesis $(\delta x-\gamma \cdot 1, y z) \in \mathscr{A} \subseteq \mathscr{A}_{1}$. Hence for all $w \in A$, $(\delta x-\gamma \cdot 1, y z, w)=0$. Since $(\gamma \cdot 1, y z, w)=0$ we get $\delta(x, y z, w)=$ $(\delta x, y z, w)=0$. Since $(\delta, p)=1$, there exist $\mu, \nu \in R$ such that $\mu p^{m}+$ $\nu \delta=1$. So $(x, y z, w)=\nu \delta(x, y z, w)=0$. Thus we have shown that

$$
(x, y, z) \neq 0 \text { implies }(x, y z, w)=0 \text { for all } w \in A \text {. }
$$

Now we proceed to show that $(x, y) \in \mathscr{A} \mathbb{A}_{1}$. For suppose not. Then $(x, y, u) \neq 0$ for some $u \in A$. Then we also have $(x, y, u+1) \neq 0$. So by (3) we get $(x, y u, w)=0=(x, y u+y, w)$ for all $w \in A$. This implies $(x, y, w)=0$ for all $w \in A$. In particular $(x, y, u)=0$, a contradiction. This contradiction shows that $(x, y) \in \mathscr{A}_{1}$. A right-left dual argument can be given to show that $(x, y) \in \mathscr{A}_{2}$. Hence $(x, y) \in \mathscr{A}$. This completes the induction step.

Lemma 3.4. Suppose $R$ is a P.I.D. and $A$ is scalar commutative. Assume fuyther that there exists a prime $p \in R, m \in Z^{+}$such that $p^{m} A=0$. Then $A$ is commutative.

Proof. Let $C$ be the center of $A$. Let $x \in A$ and $o(x)=p^{k}, k \in$ $N$. We prove by induction on $k$ that $x \in C$. If $k=0$, then $x=0$ and there is nothing to prove. So let $k>0$ and assume the 
statement for $l<k$. We first show that for any $y \in A,[x, y] \neq 0$ implies $[y x, y]=0$. So let $[x, y] \neq 0$. There exist $\alpha, \beta \in R$ such that $x y=\alpha y x$ and $(x+1) y=\beta y(x+1)$. So $\alpha y x+y=\beta y x+\beta y$. Hence $(\alpha-\beta) y x=(\beta-1) y$. If $(\alpha-\beta) y x=0$, then $\beta y=y$ and $[x, y]=0$, a contradiction. So $(\alpha-\beta) y x \neq 0$. In particular $\alpha-\beta \neq 0$ and $\alpha-\beta=p^{t} \delta$ for some $t \in N, \delta \in R$ with $(\delta, p)=1$. If $t \geqq k$, then $(\alpha-\beta) y x=0$, a contradiction. So $t<k$. Then $p^{k-t}(\beta-1) y=$ $p^{k-t}(\alpha-\beta) y x=p^{k} \delta y x=0$. Let $o(y)=p^{i}, i \in N$. If $i<k$ then by induction hypothesis $y \in C$, a contradiction. So $i \geqq k$. Hence $p^{k}\left|p^{i}\right| p^{k-t} \times$ $(\beta-1)$. So $p^{t} \mid \beta-1$ and $\beta-1=p^{t} \gamma$ for some $\gamma \in R$. So $p^{t}(\delta y x-$ $\gamma y)=0$ and by induction hypothesis $\delta y x-\gamma y \in C$. In particular $[\delta y x-\gamma y, y]=0$. Since $[\gamma y, y]=0$ we get $\delta[y x, y]=[\delta y x, y]=0$. Since $(\delta, p)=1$ there exist $\mu, \nu \in R$ such that $\mu \delta+\nu p^{m}=1$. So we get $[y x, y]=\mu \delta[y x, y]=0$. Thus we have shown that for all $y \in A$,

$$
[x, y] \neq 0 \text { implies }[y x, y]=0 .
$$

Now we proceed to show that $x \in C$. Suppose not. Then there exists $u \in A$ such that $[x, u] \neq 0$. So $[x, u+1] \neq 0$. By (4) we get $[u x, u]=$ $[u x+x, u+1]=0$. Hence $[x, u]=0$, a contradiction. This proves $x \in C$ completing the induction step.

TheOREM 3.5. Suppose $R$ is a P.I.D.

(i) If $A$ is scalar associative, then $A$ is associative.

(ii) If $A$ is scalar commutative, then $A$ is commutative.

Proof. (i) Suppose $A$ is not associative. We will get a contradiction. There exist $x, y, z \in A$ such that $(x, y, z) \neq 0$. So $(x+1$, $y, z) \neq 0$. By Lemma 3.2, $o(x y) \neq 0, o(x y+y) \neq 0$. Thus $o(y) \neq 0$. Since $(x, y+1, z) \neq 0$, the above argument shows that $o(y+1) \neq 0$. Hence $o(1) \neq 0$. Let $o(1)=d \neq 0$. Then $d$ is not a unit and hence $d=p_{1}^{i_{1}} \cdots p_{t}^{i_{t}}$ for some primes $p_{1}, \cdots, p_{t} \in A$ and some positive integers $i_{1}, \cdots, i_{t}$. Let $A_{t}=\left\{a \mid a \in A, p_{i}^{\alpha_{i}} a=0\right\}$. Then each $A_{i}$ is a nonzero subalgebra of $A$ and $A=A_{1} \oplus \cdots \oplus A_{t}$. Being subalgebras of $A$, the $A_{i}$ 's are scalar associative. Being homomorphic images of $A$, all the $A_{i}$ 's have identity elements. By Lemma 3.3, each $A_{i}$ and hence $A$ is associative, a contradiction.

(ii) Suppose $A$ is not commutative. We will obtain a contradiction. There exists $x \in A$ such that $x \notin C$, the center of $A$. So $x+1 \notin C$. By Lemma 3.2, $o(x) \neq 0$ and $o(x+1) \neq 0$. Hence $o(1) \neq 0$. By using Lemma 3.4, we obtain as in (i), that $A$ is commutative, a contradiction.

EXAMPLE 3.6. If $R$ is a field and $A$ is scalar commutative then even if $A$ does not have an identity element, Rich [4] shows that 
$A$ must be either commutative or anti-commutative. This is not true for P.I.D.'s. To see this let $A_{1}$ be the non-commutative, anticommutative algebra over $Z_{5}$ given in [4]. As a $Z$-algebra $A_{1}$ satisfies $x y=4 y x$. Now $Z_{3}$ as a $Z$-algebra satisfies the same identity. So the $Z$-algebra $A=A_{1} \times Z_{3}$ satisfies $x y=4 y x$. However $A$ is not commutative (since $A_{1}$ is not) and $A$ is not anti-commutative (since $Z_{3}$ is not).

\section{REFERENCES}

1. R. Coughlin and M. Rich, On scalar dependent algebras, Canad. J. Math., 24 (1972), 696-702.

2. R. Coughlin, E. Kleinfeld, and M. Rich, Scalar dependent algebras, Proc. Amer. Math. Soc., 39 (1973), 69-73.

3. N. H. McCoy, The Theory of Rings, New York, Macmillan, 1964.

4. M. Rich, A commutativity theorem for algebras, Amer. Math. Monthly, 82 (1975), 377-379.

Received November 13, 1975 and in revised form February 11, 1976.

North Carolina State University at Raleigh 


\title{
PACIFIC JOURNAL OF MATHEMATICS
}

\author{
EDITORS
}

\author{
RICHARD ARENS (Managing Editor) \\ University of California \\ Los Angeles, California 90024
}

\author{
R. A. Beaumont \\ University of Washington \\ Seattle, Washington 98105
}

J. DugundjI

Department of Mathematics

University of Southern California

Los Angeles, California 90007

D. Gilbarg and J. Milgram

Stanford University

Stanford, California 94305

\section{ASSOCIATE EDITORS}
E. F. BECKENBACH
B. H. NEUMANN
F. WOLF
K. Yoshida

\section{SUPPORTING INSTITUTIONS}

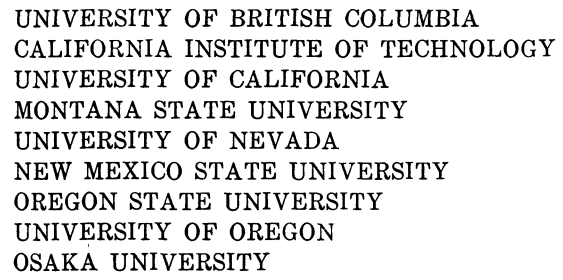

UNIVERSITY OF BRITISH COLUMBIA CALIFORNIA INSTITUTE OF TECHNOLOGY UNIVERSITY OF CALIFORNIA MONTANA STATE UNIVERSITY UNIVERSITY OF NEVADA NEW MEXICO STATE UNIVERSITY OREGON STATE UNIVERSITY UNIVERSITY OF OREGON OSAKA UNIVERSITY

\author{
UNIVERSITY OF SOUTHERN CALIFORNIA \\ STANFORD UNIVERSITY \\ UNIVERSITY OF HAWAII \\ UNIVERSITY OF TOKYO \\ UNIVERSITY OF UTAH \\ WASHINGTON STATE UNIVERSITY \\ UNIVERSITY OF WASHINGTON

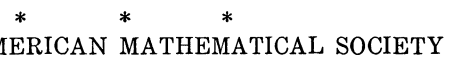

The Supporting Institutions listed above contribute to the cost of publication of this Journal, but they are not owners or publishers and have no responsibility for its content or policies.

Mathematical papers intended for publication in the Pacific Journal of Mathematics should be in typed form or offset-reproduced, (not dittoed), double spaced with large margins. Please do not use built up fractions in the text of your manuscript. You may however, use them in the displayed equations. Underline Greek letters in red, German in green, and script in blue. The first paragraph or two must be capable of being used separately as a synopsis of the entire paper. Items of the bibliography should not be cited there unless absolutely necessary, in which case they must be identified by author and Journal, rather than by item number. Manuscripts, in triplicate, may be sent to any one of the editors. Please classify according to the scheme of Math. Reviews, Index to Vol. 39. All other communications should be addressed to the managing editor, or Elaine Barth, University of California, Los Angeles, California, 90024.

The Pacific Journal of Mathematics expects the author's institution to pay page charges, and reserves the right to delay publication for nonpayment of charges in case of financial emergency.

100 reprints are provided free for each article, only if page charges have been substantially paid. Additional copies may be obtained at cost in multiples of 50 .

The Pacific Journal of Mathematics is issued monthly as of January 1966. Regular subscription rate: $\$ 72.00$ a year (6 Vols., 12 issues). Special rate: $\$ 36.00$ a year to individual members of supporting institutions.

Subscriptions, orders for back numbers, and changes of address should be sent to Pacific Journal of Mathematics, 103 Highland Boulevard, Berkeley, California, 94708.

PUBLISHED BY PACIFIC JOURNAL OF MATHEMATICS, A NON-PROFIT CORPORATION

Printed at Kokusai Bunken Insatsusha (International Academic Printing Co., Ltd.), 8-8, 3-chome, Takadanobaba, Shinjuku-ku, Tokyo 160, Japan.

Copyright (C) 1975 by Pacific Journal of Mathematics Manufactured and first issued in Japan 


\section{Pacific Journal of Mathematics}

\section{Vol. 63, No. 2 \\ April, 1976}

Joseph Anthony Ball and Arthur R. Lubin, On a class of contractive perturbations

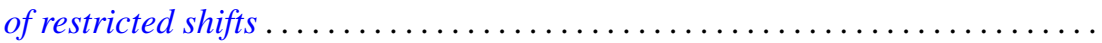

Joseph Becker and William C. Brown, On extending higher derivations generated

by cup products to the integral closure .......................

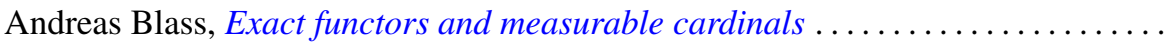

Joseph Eugene Collison, A variance property for arithmetic functions . . . . . . . . . .

Craig McCormack Cordes, Quadratic forms over nonformally real fields with a

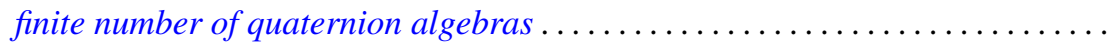

Freddy Delbaen, Weakly compact sets in $H^{1} \ldots \ldots \ldots \ldots \ldots \ldots \ldots \ldots \ldots$

G. D. Dikshit, Absolute Nörlund summability factors for Fourier series ..........

Edward Richard Fadell, Nielsen numbers as a homotopy type invariant. . ........

Josip Globevnik, Analytic extensions of vector-valued functions . . . . . . . . . . . .

Robert Gold, Genera in normal extensions . . . . . . . . . . . . . . . . . . . 389

Solomon Wolf Golomb, Formulas for the next prime

Robert L. Griess, Jr., The splitting of extensions of $S L(3,3)$ by the vector space

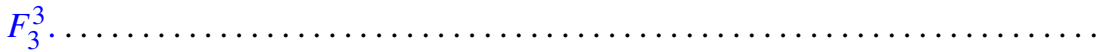

Thomas Alan Keagy, Matrix transformations and absolute summability .........

Kazuo Kishi, Analytic maps of the open unit disk onto a Gleason part.

Kwangil Koh, Jiang Luh and Mohan S. Putcha, On the associativity and commutativity of algebras over commutative rings . ..... . .

James C. Lillo, Asymptotic behavior of solutions of retarded differential difference equations.

John Alan MacBain, Local and global bifurcation from normal eigenvalues ..

Anna Maria Mantero, Sets of uniqueness and multiplicity for $L^{p}$

J. F. McClendon, Embedding metric families

L. Robbiano and Giuseppe Valla, Primary powers of a prime ideal .

Wolfgang Ruess, Generalized inductive limit topologies and barrelledness

properties.

Judith D. Sally, Bounds for numbers of generators of Cohen-Macaulay ideals

Helga Schirmer, Mappings of polyhedra with prescribed fixed points and fixed point indices.

Cho Wei Sit, Quotients of complete multipartite graphs

S. Sznajder and Zbigniew Zielezny, Solvability of convolution equations in $\mathscr{K}_{p}^{\prime}$,

$p>1$.

Mitchell Herbert Taibleson, The existence of natural field structures for finite

dimensional vector spaces over local fields

William Yslas Vélez, A characterization of completely regular fields

P. S. Venkatesan, On right unipotent semigroups ..............

Kenneth S. Williams, A rational octic reciprocity law ............

Robert Ross Wilson, Lattice orderings on the real field .......... 